\title{
Virtual Preoperative Simulation for Excision of Spinal Tumors: Surgeon Processing of Medical Computer-Assisted Design Software
}

\author{
Jung Hwan Lee ${ }^{1}$, \\ In Ho Han', \\ Byung Kwan Choi ${ }^{1}$, \\ Kyoung Hyup Nam", \\ Dong $\mathrm{Ha} \mathrm{Kim}{ }^{2}$, \\ Chi Seung Lee ${ }^{3}$
}

${ }^{1}$ Department of Neurosurgery, Pusan National University Hospital, Busan, ${ }^{2}$ Department of Neurosurgery, Dong-Eui Medical Center, Busan, ${ }^{3}$ Biomedical Research Institute, Pusan National University Hospital, Busan, Korea

\section{Corresponding Author: \\ In Ho Han}

Department of Neurosurgery, Pusan National University Hospital, Pusan National University School of

Medicine, 179 Gudeok-ro, Seo-gu, Busan 49241, Korea

Tel: +82-51-240-7257

Fax: +82-51-244-8373

E-mail: farlateral@hanmail.net

Received: September 3, 2017 Revised: October 10, 2017 Accepted: October 11, 2017
Copyright $\left({ }^{\circ} 2017\right.$ by The Korean Spinal Neurosurgery Society

This is an open access article distributed under the terms of the Creative Commons Attribution Non-Commercial License (http://creativecommons.org/licenses/by-n c/4.0/) which permits unrestricted non-commercial use, distribution, and reproduction in any medium, provided the original work is properly cited.

As medical computer-aided design (CAD) has improved, virtual 3-dimensional medical images have been gaining more easily without any special practice. These images can be applied to various clinical fields. This article illustrates virtual preoperative simulation for excision of spinal tumors using medical CAD software. The software was used directly by the surgeon. The process of virtual preoperative simulation for spinal tumor surgery was found to be not inordinately complicated. And, virtual simulation was helpful in determining surgical steps as well as understanding the surgical anatomy.

Key Words: Three-dimensional imaging, Planning technique, Spinal cord neoplasms, Computer-aided design

\section{INTRODUCTION}

Computer-aided design (CAD) software has been applied to the automobile and aerospace industries since the 1970s. CAD increased productivity with predictable outcomes. As a result of its initial success, use of CAD technology has been widely spread, and applied to various other industries. In parallel, computed tomography (CT) scans have developed enough to represent the 3-dimensional (3D) structure of the living human body ${ }^{4)}$. Advances in these technologies have resulted in the creation of virtual medical imaging and the medical CAD software to process it $^{8)}$. There are many examples of clinical practices using this software in various surgeries ${ }^{6,8)}$. The applications extended from customized implants production to making intraoperative guidance ${ }^{5,7}$. There have also been various attempts to apply this software to spinal surgery ${ }^{5,6}$. Initially, such software was difficult to handle and required special training. However, the development of medical CAD software allows the surgeon to preview all the necessary surgical steps directly ${ }^{6}$. This article illustrates virtual preoperative simulation for excision of spinal tumors via medical CAD software, as used directly by a spinal surgeon.

\section{TECHNICAL NOTE}

The entire processing of $\mathrm{CAD}$ for virtual simulation of spinal tumor excision was performed by a spinal surgeon. Fine CT images of the spine were obtained. The thickness of each slice was $0.625 \mathrm{~mm}$. Mimics 17.0 (Materialise $\mathrm{NV}$, Leuven, Belgium) software was used for image editing and visualization. CT scans were imported into digital imaging and communications in medicine (DICOM) format. To start the import wizard, the user first selects 'File' and then chooses 'New Project Wizard.' In the File Browser window, the surgeon can select where the images to be imported are found. The user then clicks the 'Next' button to proceed to the Studies window, and presses the 'Convert' button (Fig. 1A). If the image orientations are correct, the user then clicks the 'OK' button.

To change the threshold value, the 'Threshold' button is used. A low threshold value makes it possible to select soft tissue. Convertsely, in high threshold values, dense and enhanced parts were selected (Fig. 1B). Each selected tissue was registered as a 'mask' of a different color. Using the 'edit mask' button, some bony structures were hidden to determine the extent of bone resection necessary to ensure that the tumor was fully exposed. Each organ (major vessels, bony structures, thecal sac and tumor) were distinguished by their respective colors (Fig. 1C). Then, the 'Calculate 3D' button is pressed to convert each mask into a 3D image. Reconstructed surgical field were rotated at various angles to find proper approaches for surgery (Fig. 1D). Additionally, the organs covering the tumor could be hidden to confirm the surgical steps required for tumor removal. And, the tumor also was hidden for confirmation of surrounding structures after totally tumor removal. 


\section{CASE REPORTS}

\section{Case 1: Large Schwannoma of the Sacrum}

A 33-year-old female was transferred to Pusan National University Hospital due to sciatica. She had no documented medical history. Magnetic resonance image (MRI) scans demonstrated a well enhanced mass on the left side of the sacrum (Fig. 2A). A percutaneous biopsy was done and the pathologic diagnosis was found to be schwannoma. We planned total surgical resection. CT scans were obtained and virtual 3D model was reconstructed using Mimics (Fig. 2B, C). The extent of the laminectomy was determined (Fig. 2F). Bony margin after total tumor removal was also confirmed (Fig. 2G). During the actual surgery, the laminectomy was performed as planned, and the tumor was fully exposed (Fig. 2D, E). The tumor was removed entirely, and there were no surgery-related complications.

\section{Case 2: Malignant Peripheral Nerve Sheath Tumor of the Thoracic Spine}

A 40-year-old male visited Pusan National University Hospital due to back pain and leg weakness. He had been previously diagnosed with neurofibromatosis. MRI scans of the spine revealed a well-enhanced mass on the thoracolumbar junction. The tumor eroded the vertebral body and extended into the pleura (Fig. 3A). CT scans were obtained and preoperative virtual planning was performed in the same method as described above (Fig. 3B, D-G). The extent of rib excision and laminectomy was determined (Fig. 3B, F). The surgery proceeded as planned virtually (Fig. 3C). The level of tumor exposure was similar to the virtual image (Fig. 3B, C). There were no surgery-related complications, and the patient achieved rehabilitation from leg weakness.

\section{DISCUSSION}

Conventional preoperative planning has limits for accurate simulation of real anatomic structure when based on 2-dimensional (2D) radiographic images ${ }^{6}$. For many patients, advanced $3 \mathrm{D}$ imaging and use of medical CAD software can improve the accuracy of treatment planning and the precision of surgical execution $^{6}$. These types of software could also be potentially applied to intraoperative guidance, real-time navigation and patient-customized implants ${ }^{6,7)}$.

Virtual preoperative simulation using CAD software has been applied to various surgical areas. In particular, many authors have attempted to apply it to oral and maxillofacial surgery ${ }^{6-8)}$. Their measure of success was determined not only by functional outcomes but cosmetic outcomes as well. Therefore, there has been a lot of interest in this technique among oral and max-

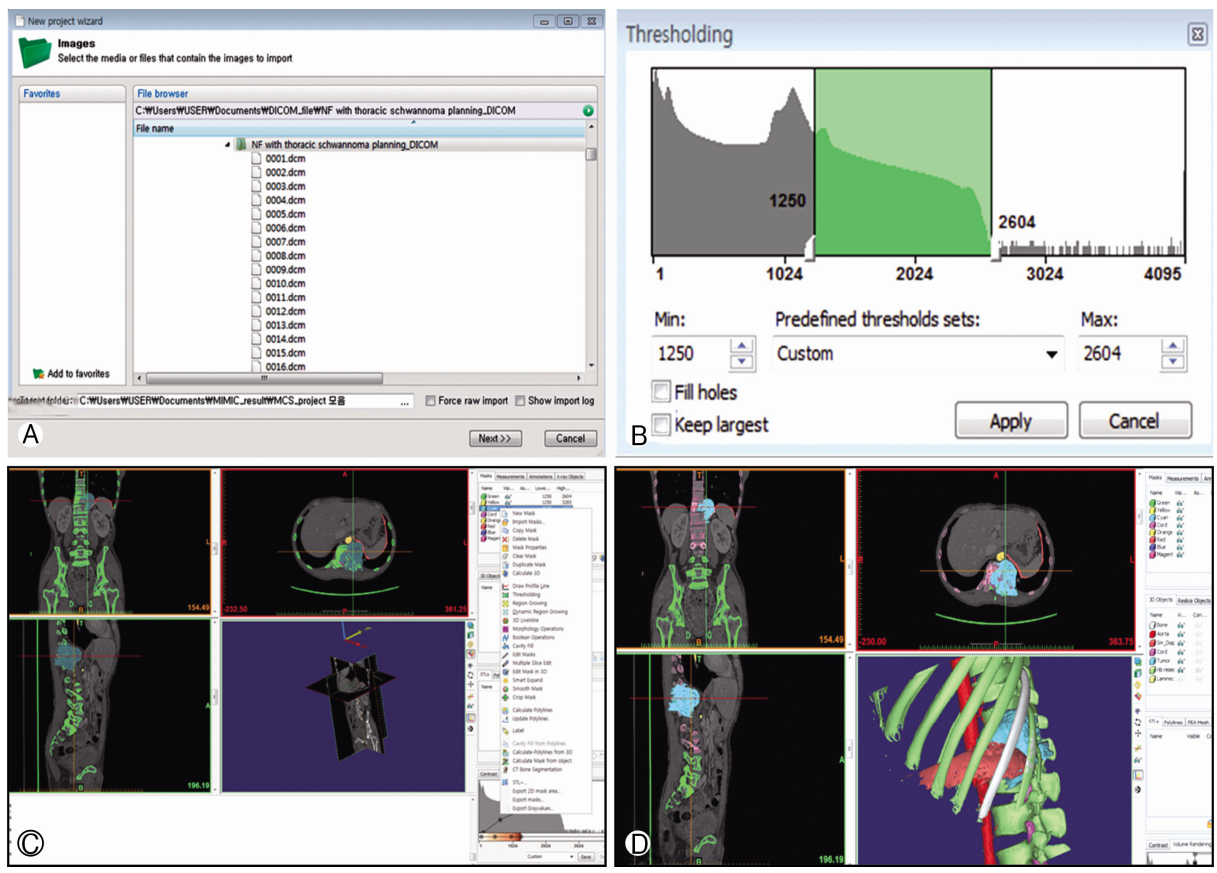

Fig. 1. (A) A window for selecting digital imaging and communications in medicine (DICOM) files. Select the folder containing DICOM files and click the 'next' button to proceed automatically. (B) A window for thresholding. The higher the threshold value, the more dense and enhanced the part selected. (C) Various colored masks representing different organs. Using the 'edit mask' button, some images could be hidden for confirmation of proper exposure. (D) Three-dimensional reconstruction of each mask. It was possible to identify proper surgical exposure through hiding some organ or planned bone resection areas. 

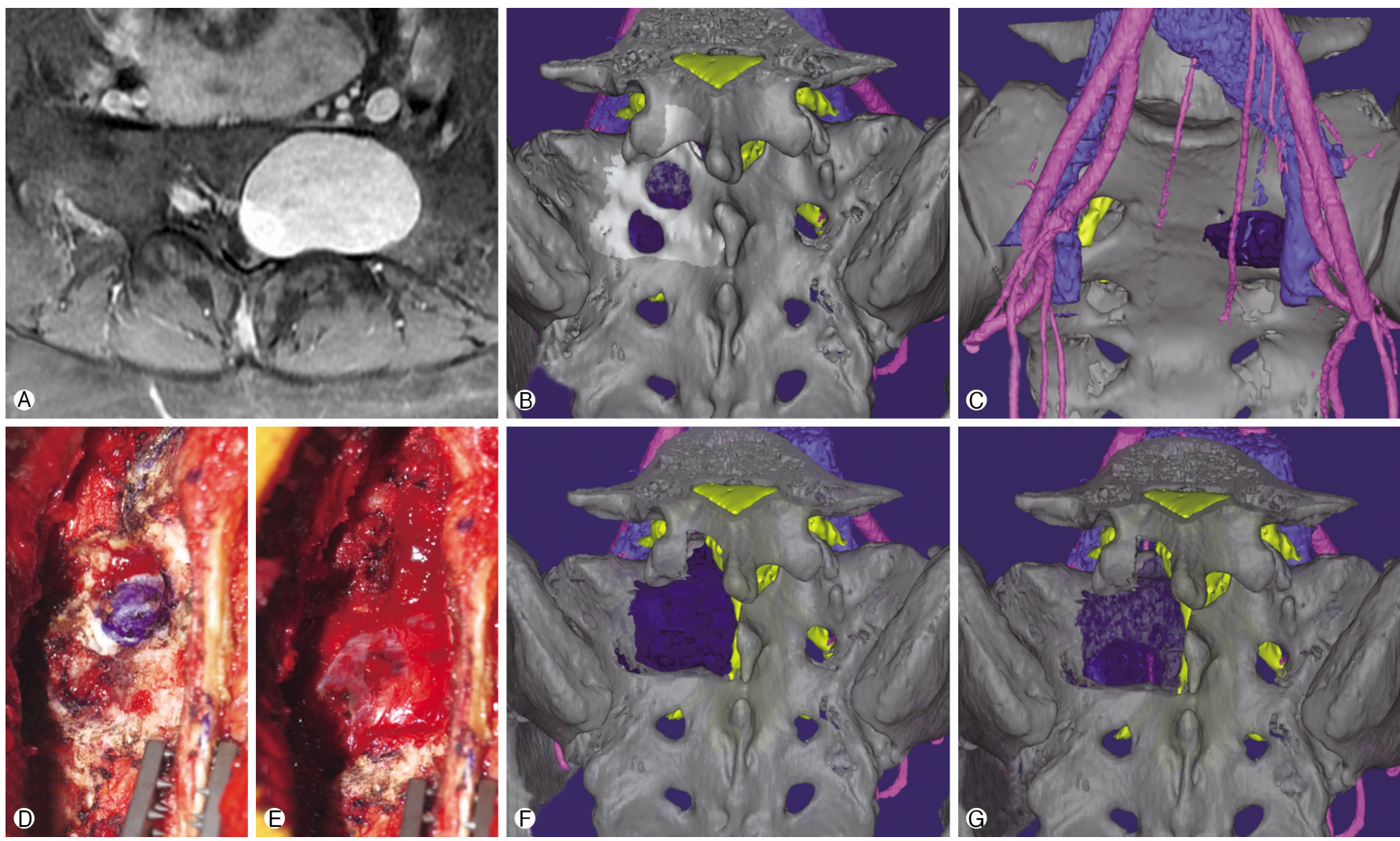

Fig. 2. Case 1. (A) Magnetic resonance image scans revealed a well-enhanced mass on the left side of the sacrum. (B) Three-dimensional (3D) reconstruction (posterior view). (C) 3D reconstruction (anterior view). (D) During the actual surgery, we marked the place where the laminectomy would be performed. (E) After the laminectomy, the tumor was exposed fully. (F) Extent of laminectomy in which the tumor was fully exposed. (G) After tumor removal, the surrounding anatomy was confirmed.

illofacial surgeons. In spine surgery, previous attempts have also been made to apply virtual preoperative simulation ${ }^{1)}$. Archavlis et al. ${ }^{1)}$ reported some cases of successful transpedicular corpectomy planned by $3 \mathrm{D}$ reconstruction software. Lin et al. ${ }^{5}$ reported some invasive sacral schwannoma treated by customized osteotomy. In particular, cases of unfamiliar anatomical areas, such as the sacrum, complex neurovascular structure or high cervical area surgery, virtual preoperative simulation could provide more useful information than simple 2D images or limited CT-based 3D reconstruction. For years, it took special and long training sessions to learn how to use such software. Therefore, generally speaking, cooperation with biomedical engineers has been necessary ${ }^{2,3,5)}$.

However, this cooperation was not always successful. Some intraoperative guidance or patient-customized implants made by biomechanical engineers were found to be unsuitable for surgery. The suspected reason for this was that the engineers were not well aware of the surgical procedure and therefore could not accurately reflect the intentions of the surgeon. Preoperative simulation has numerous variables that engineers with no experience in surgery could not understand. Additionally, preoperative simulation was not a process in which special products were produced. As a result, biomechanical engineers became less inter- ested in preoperative simulation because it had no foreseeable commercial value. Also, cooperation with engineers was not possible for all cases of surgery. Therefore, simulations using medical CAD software should be implemented by the actual user.

Handling this software directly has another advantage. Recently, the performance of 3D printers has been getting better, and the price cheaper. This allows for individual use of the 3D printers. Using a 3D printer, a surgeon could directly create intraoperative guidance or other devices to help the surgery. To do this, however, the surgeon should be accustomed to making the design for an output of a 3D printer. In this process, the proper and direct handling of CAD software is essential.

As mentioned earlier, $\mathrm{CAD}$ software was difficult to use. To convert DICOM files to the standard 3D image format, another piece of software such as ScanIP (Synopsys Inc., Mountain View, CA, USA) or Osirix (Pixmeo SARL, Geneva, Switzerland) should be used. However, with recent advancements in medical CAD software, this software has become more intuitive and simpler. In addition to Mimics we used, Dolphin Imaging (Dolphin Imaging and Management Solutions, Chatsworth, CA, USA) and Maxilim (Medicim, Mechelen, Belgium) were known as medical CAD software $^{6}$. These programs did not take an exceedingly long time to perform preoperative simulations. In the context of our cases, the 

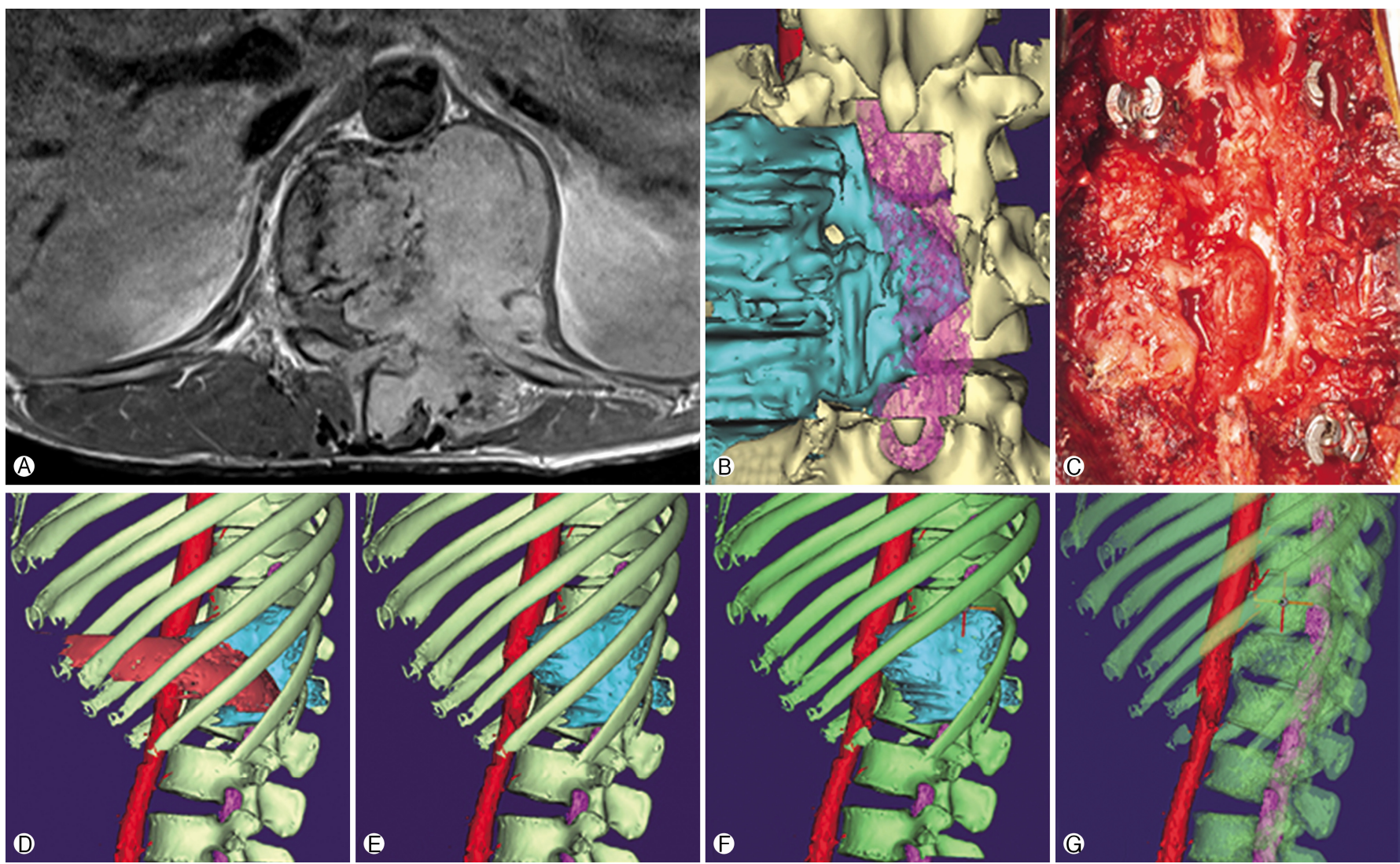

Fig. 3. Case 2. (A) Magnetic resonance image scans showed a well-enhanced mass. The mass invaded the vertebral body and extended into the pleura. (B) Three-dimensional (3D) reconstruction (posterior view) with virtual laminectomy. (C) During the actual surgery, the tumor was exposed fully after performing the laminectomy as planned virtually. (D) 3D reconstruction (lateral view). (E) The diaphragm was hidden in order to allow exposure of tumor. (F) The 11th rib was hidden. It could be virtually confirmed that removing this rib only was enough to expose the tumor. (G) The tumor was hidden thereby confirming the surrounding normal organs.

first case took about 60 minutes and the second case about 45 minutes. This time would potentially be shorter if the software was accustomed. Therefore, surgeons alone could make enough virtual preoperative simulation. Based on these technical notes, we conclude that virtual preoperative simulation using medical $\mathrm{CAD}$ software direc- tly could be helpful in predicting the actual surgical procedures.

This technique had some limitations. At first, Mimics was paid software, and expensive for the personal user. Therefore, accessibility was limited except for those commercially licensed to design medical products. In our cases, we performed these processes using software purchased from a division of biomechanics as under an academic license. Also to be considered was the fact, fine CT scans were necessary. In conventional CT images, the quality of the 3D image is comparatively deteriorated and it may be difficult to analyze. Additional CT scans increase total cost and exposure of radiation to the patient.

\section{CONCLUSION}

Virtual preoperative simulation using medical CAD software has the potential to optimize various surgical options. Recently, the user interface of medical CAD software has been more intuitive and easy to use. Therefore, we conclude that it is advantageous for the surgeon to perform virtual preoperative simulation using medical CAD software directly when preparing for the excision of a spinal tumor.

\section{CONFLICT OF INTEREST}

No potential conflict of interest relevant to this article was reported.

\section{REFERENCES}

1. Archavlis E, Schwandt E, Kosterhon M, Gutenberg A, Ulrich P, Nimer A, et al: A modified microsurgical endoscopic-assisted transpedicular corpectomy of the thoracic spine based on virtual 3-dimensional planning. World Neurosurg 91:424-433, 2016

2. Chen X, Cheng J, Gu X, Sun Y, Politis C: Development of preoperative planning software for transforaminal endoscopic surgery and the guidance for clinical applications. Int J Comput Assist Radiol Surg 11:613-620, 2016 
3. Hicdonmez T, Parsak T, Cobanoglu S: Simulation of surgery for craniosynostosis: a training model in a fresh cadaveric sheep cranium. Technical note. J Neurosurg 105(2 Suppl):150-152, 2006

4. Hounsfield GN: Computerized transverse axial scanning (tomography). 1. Description of system. Br J Radiol 46:1016-1022, 1973

5. Lin CL, Fang JJ, Lin RM: Resection of giant invasive sacral schwannoma using image-based customized osteotomy tools. Eur Spine J 25:4103-4107, 2016

6. Lin HH, Lo LJ: Three-dimensional computer-assisted surgical simu- lation and intraoperative navigation in orthognathic surgery: a literature review. J Formos Med Assoc 114:300-307, 2015

7. Metzger MC, Hohlweg-Majert B, Schwarz U, Teschner M, Hammer B, Schmelzeisen R: Manufacturing splints for orthognathic surgery using a three-dimensional printer. Oral Surg Oral Med Oral Pathol Oral Radiol Endod 105:e1-7, 2008

8. Zhao L, Patel PK, Cohen M: Application of virtual surgical planning with computer assisted design and manufacturing technology to cranio-maxillofacial surgery. Arch Plast Surg 39:309-316, 2012 